\title{
Interactive Heart-Rate Pulse Printer
}

\author{
Kang-Hee Lee \\ Global School of Media, Soongsil University, \\ Seoul 156-743, Republic of Korea \\ E-mail: kanghee.lee@ssu.ac.kr
}

\begin{abstract}
This paper proposes an interactive media artwork called the Heart-Rate Pulse Printer. The proposed artwork measures a participant's heart rate directly through a real heartrate pulse-sensor and shows a reproduction of his/her original pulse graphed by LED. This Heart-rate Pulse Printer captures an inner moment in time that cannot be recreated. The experimental results verify the feasibility of the proposed artwork.
\end{abstract}

Keywords: Pulse Sensor, Camera, Pulse Printer

\section{Introduction}

An afterimage is a non-specific term that refers to an image continuing to appear in one's vision after the exposure to the original image has ceased [1]. An afterimage is a normal phenomenon (physiological afterimage) or it may sometimes be pathological. A common physiological afterimage is the dim area that seems to float before one's eyes after briefly looking into a light source, such as a flash from a digital camera. Due to the wide spread of digital cameras and rapid development of the mobile devices with cameras, we are able to take pictures at any moment and place [2]. People enjoy taking pictures and sharing them with others, and they care very much about their appearances in those pictures. Even when people look beautiful in the pictures, their internal images are not expressed [3]. Thus, we would like to develop photographs of the inner feelings, as shown in Figure 1.

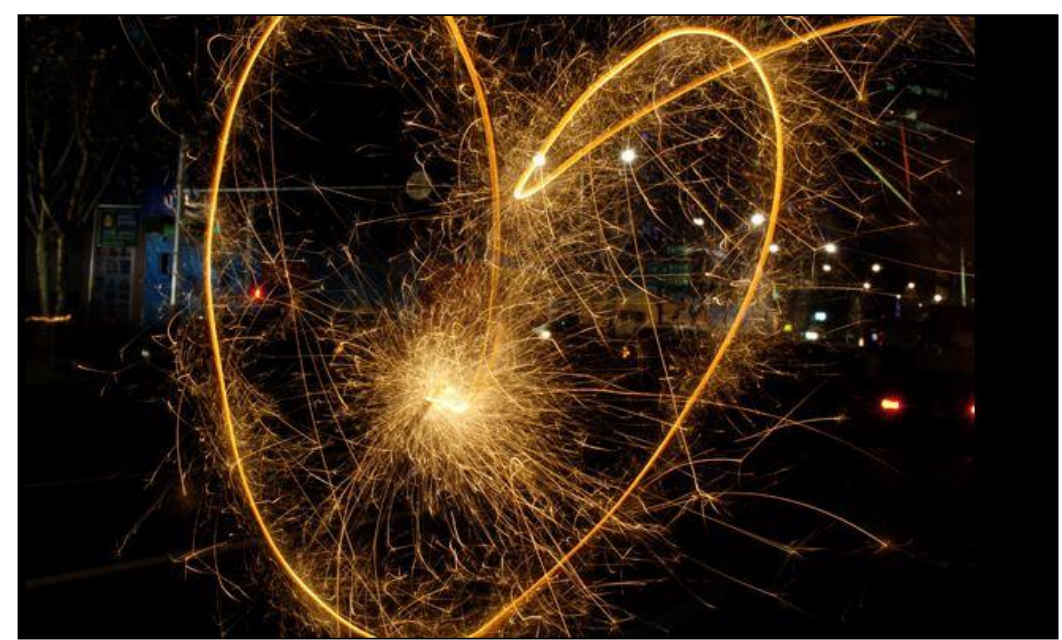

Figure 1. The Expression of the Emotion, 'LOVE', using the Light Afterimage Captured with Low Shutter Speed

The LED installation artistically invites the visitors to relax in a turquoise beanbag chair while taking in a dazzling light show on an enormous screen. Though it's tempting 
to search for a pattern, a meaning, or a message in this light show, the only true meaning comes from what the individual brings to the piece. Whether or not Villareal intended a message, the installation can be enjoyed purely for its aesthetic value. The dancing patterns of alternating sets in different shades of magenta, teal, red, yellow, and blue encourage viewers to sit and unwind [4]. In reality, there are many commercial or artistic products employing LED afterimages, such as a LED globe [5], a propeller LED clock [6], and a universal LED bar [7].

Generally, we imagine the heart as a mediator representing our mind. We can express the excitement and nervousness that we feel in our minds by showing the movement of the heart rather than by showing the facial expressions. Also, by letting people manipulate the image in the camera, they will realize that this image is another expression of them. The pulse sensor is used to measure the heart rate of the spectator. The shutter speed of the camera is so low that the heart rate may be graphed by LED, as shown in Figure 1. Finally, the media art could be experienced by the spectator.

The remaining structure of this paper is outlined as follows. Section 2 briefly introduces our pulse printer system and its methodology. The new concept of using the interactive pulse printer as a source for media art is proposed. The process of the pulse printer consists of measuring, expressing, and capturing the pulse of a user's heart rate. Section 3 describes in detail the ways to implement the pulse printer. Section 4 describes the experiments carried out to demonstrate its performance. The empirical and theoretical analyses follow to investigate the characteristics of the pulse printer. Section 5 is composed of concluding remarks.

\section{Concept of Heart-Rate Pulse Printer System}

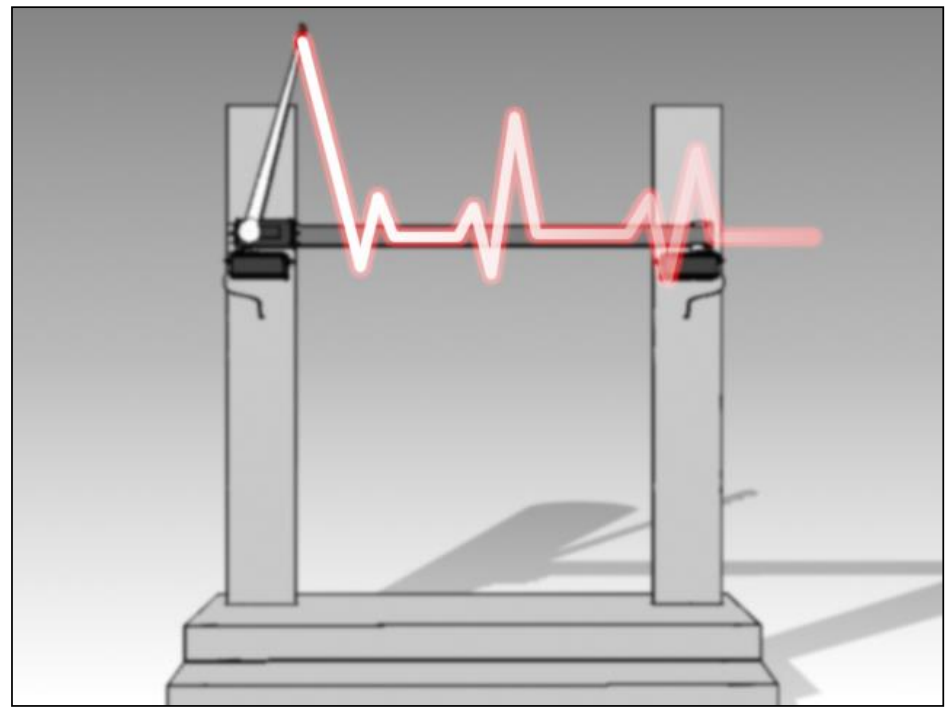

Figure 2. The Physical Concept of the Heart-Rate Pulse Printer

The heart-rate pulse printer system is composed of three parts: the pulse sensor that measures a spectator's heart-rate, the motors that displays the measured pulse input, and the DSLR camera that captures the displayed LED afterimage. There are two kinds of motors; one is responsible for the horizontal movement called stepper motor, and the other is servo motor which is responsible for the vertical movement. The motors obtain the figures through the sensor, and the speed and the angle of the servo motor are adjusted. After a horizontal movement, the vertical motion draws a graph of the heart rate. The camera films the spectator's heart rate. This time by setting the camera's shutter speed to low, the graph represents the after image of the LED. 


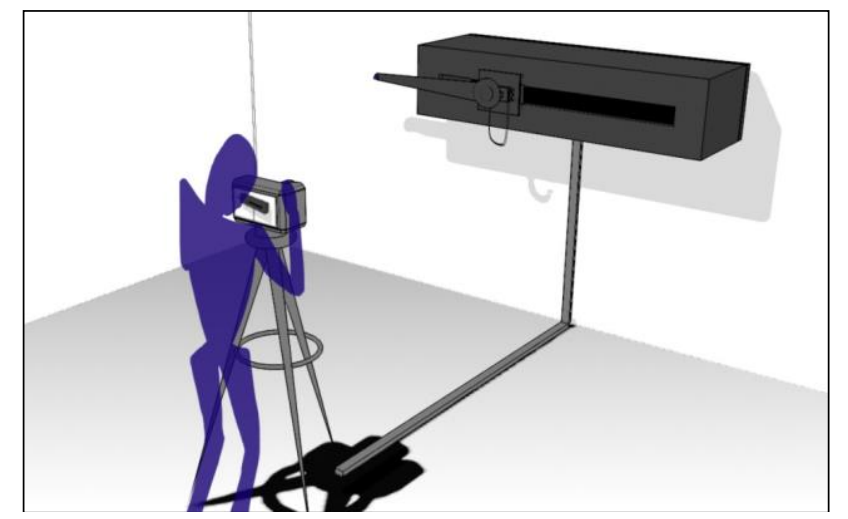

Figure 3. The Concept of Appreciating the Interactive Heart-Rate Pulse Printer

\section{Design and Implementation}

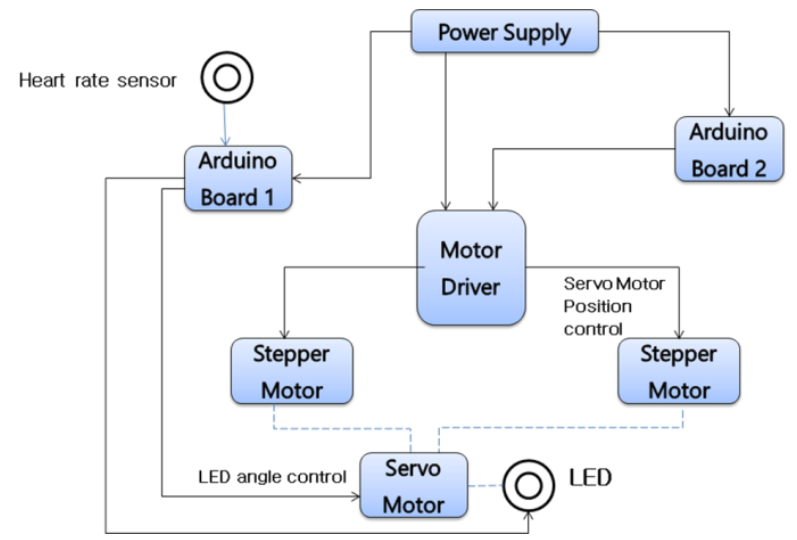

Figure 4. Overall Hardware Structure

This artwork operates with the power supply to Arduino controller boards and two motors, as shown in Figure 4. In front of the artwork, there must be a camera and the shutter speed should be set to less than 8 seconds. The person needs to wear the heart rate sensor that is connected to the artwork and shoot the graph that is drawn by the artwork, as shown in Figures 3 and 4.

\subsection{Control Part}

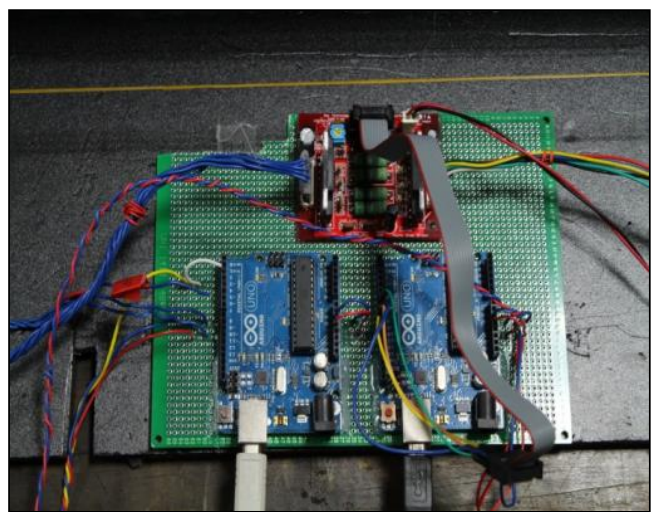

Figure 5. Implementation of the Heart-Rate Pulse Printer Controller Circuit 
As shown in Figure 5, two microcontrollers called Arduino were used. One is for controlling the stepper motor and processing the input from heart rate sensor. The other is for controlling the servo motor. As shown in Figures 4 and 5, controlling the stepper motor requires a separate motor driver.

\subsection{Measuring and Capturing Part}

This part measures the heart rate of the spectator. The sensor is an important element in the system by itself (Figure 6) or in combination with other systems such as cameras (Figure 7).

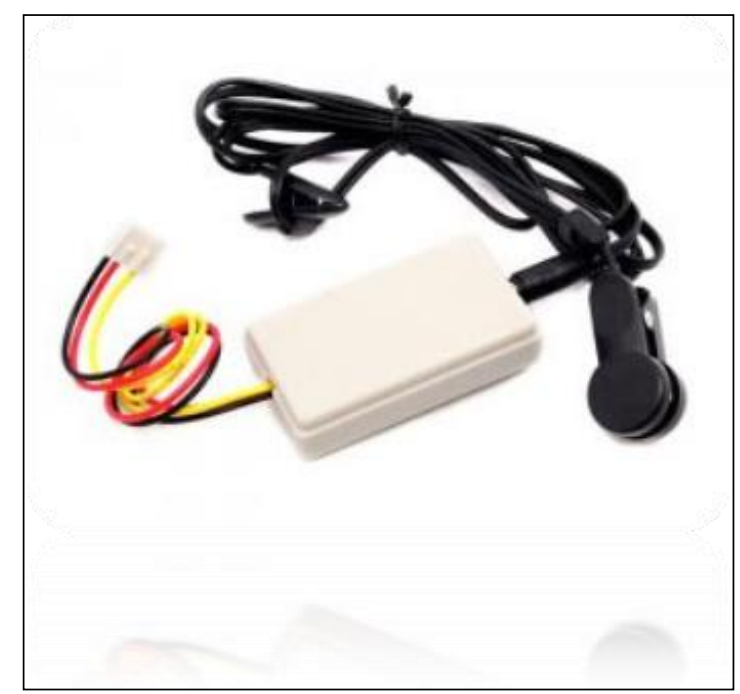

Figure 6. Ear/Finger-Clip Heart-Rate Sensor

The ear-clip heart-rate sensor can be used by itself as a pulse sensor, as shown in Figure 6. It contains an ear clip and a white-box receiver module. It has high sensitivity and low power consumption. It is very convenient, portable (Model name: MED03212P), and operates in a wide range of power supply- DC 3 5 V. In this optical heart-rate pulse sensor, light is passed through a fingertip or ear lobe. The light (infrared diode) either bounces back to the light sensor (photodiode) or becomes absorbed into the blood cells. When the spectator clips this sensor on the ear lobe, the result is transferred to the arduino board 1 via the serial port. The result is saved for analysis, and it generates the angle of the LED.

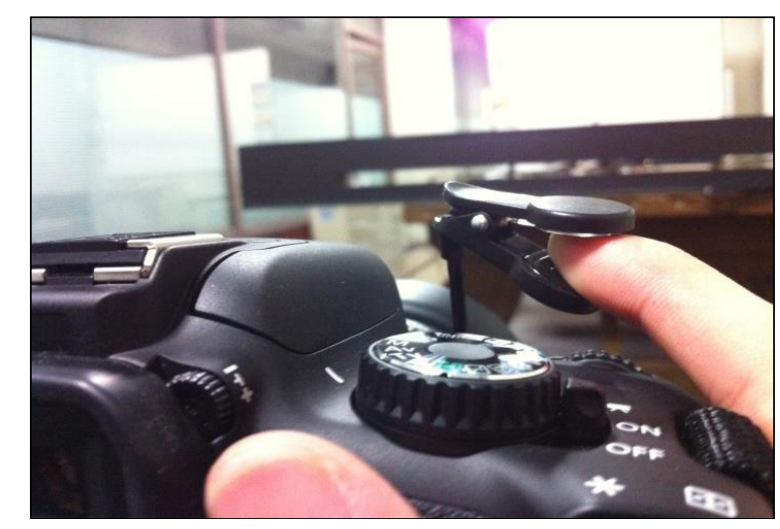

Figure 7. The Capturing Part of the Heart-Rate Pulse Printer Using FingerTipped Pulse Sensor 


\subsection{Manipulation Part}

This part reproduces the measured heart rate with a servo motor and a stepper motor. As shown in Figure 8, the servo motor is responsible for the vertical angular motion, while the stepper motor is for the horizontal reciprocating motion.
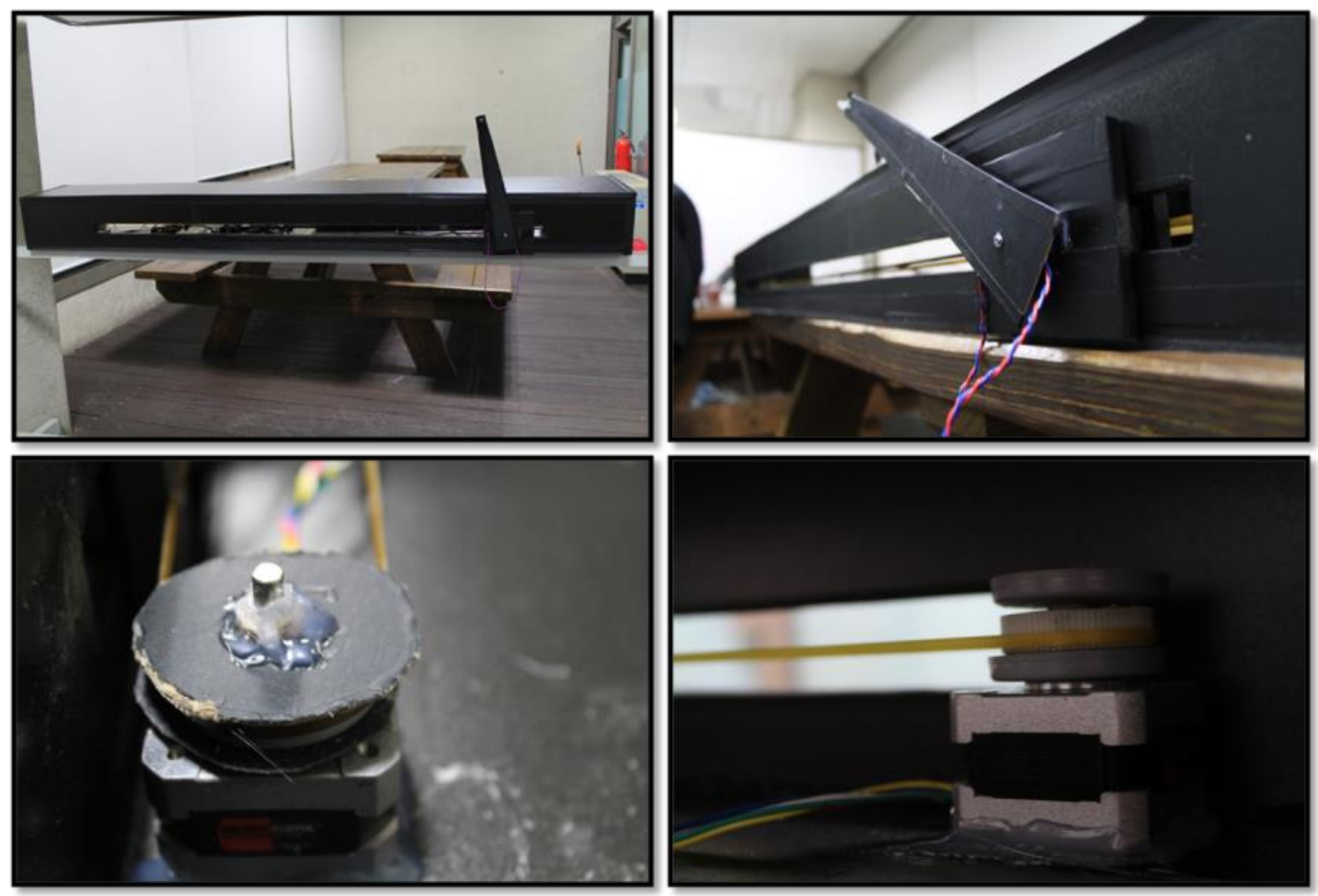

Figure 8. The Stepper Motor for Horizontal Movement of LED-Head and the
Servo Motor for Vertical Movement of LED-Head

The servo motor (HS-430BH) operates between $4.8 \mathrm{~V}$ and $6.0 \mathrm{~V}$. Its speed is 0.10 $\mathrm{sec} / 60$ degree and the torque is $1.4 \mathrm{~kg}-\mathrm{cm}$. The LED-mounted printer head continues to move vertically while this servo motor makes a little noise. The detailed specification of stepper motor (NK243-01AT) is as follows. It is unipolar-type and has one winding with center tap per phase. It has the resolution of $1.8 \%$ step, the current of $0.95 \mathrm{~A} / \mathrm{phase}$, the holding torque of $1.6 \mathrm{~kg}-\mathrm{cm}$, the detent torque of $120 \mathrm{~g}$ $\mathrm{cm}$, the rotor inertia of $38 \mathrm{~g}-\mathrm{cm}$, and the weight of $200 \mathrm{~g}$. The belt is highly elastic, and the belt pulley is recycled with the one from a broken inkjet printer.

The stepper motor makes the LED move from side to side on the rail. Basically, it starts from the right side. After ending on the left side, it returns to the starting point without vertical motion. The length of the guidance rail is $1 \mathrm{~m}$, and its operation holds between 10 and 20 seconds. Even if the pulse is not measured, the horizontal motion is effective, because it can still express the state of death.

\section{Experiments}

This section measured the state of hearts from different spectators and reproduced their state into the proposed artwork. Figure 9 shows the flowchart of the proposed system. 


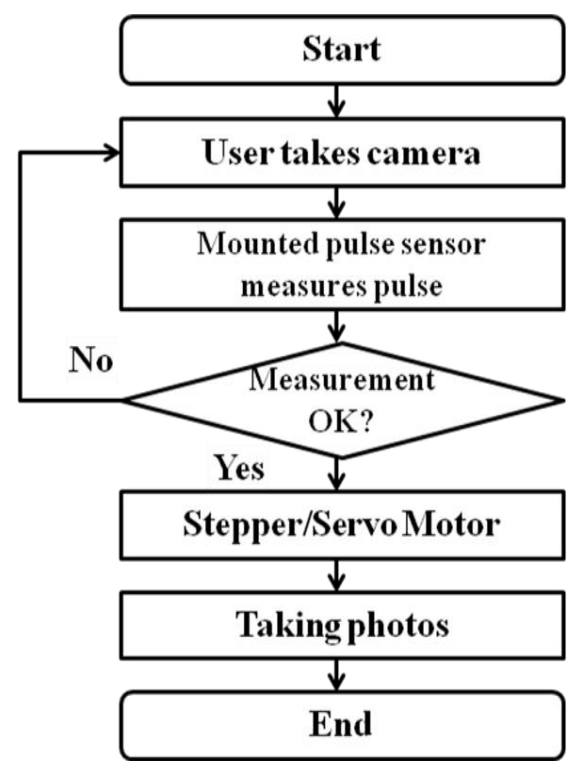

Figure 9. Flowchart of the Overall System

First, a spectators or a user takes the camera, on which the pulse sensor is mounted. Second, the pulse sensor measures the spectator's heart rate. Third, the measured pulse rate is recreated by the movement of the LED mounted on a stepper motor and a servo motor. In real time, the angle of the servo motor depends on the heart-rate of the spectator, and it becomes the motive power by which his/her emotion can be shared with others.

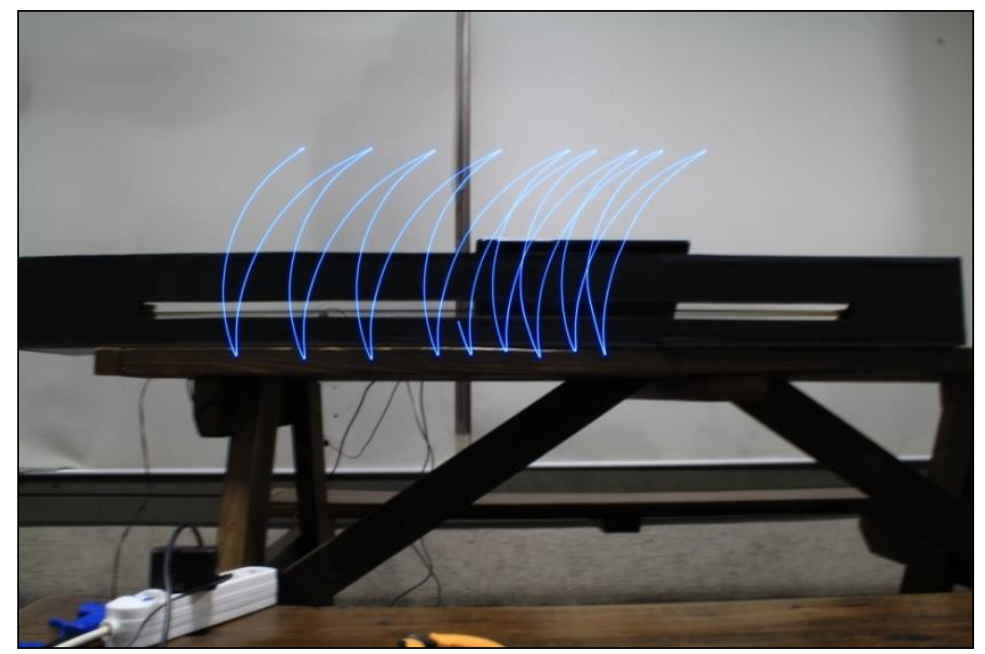

Figure 10. The Heart Rate Graphed by LED Mounted on the Step Motor

Fourth, since the DSLR camera is set up with a low shutter speed, it captures the overall afterimage trajectory of LED, as shown in Figure 10. Finally, the spectator can have his/her own four dimensional graph of the heart rate.

While the servo motor ranges from -90 to 90 degrees, the heart rate ranges from 0 to 250. To solve this difference, the mapping function in Arduino controller is used effectively as shown in Figure 10. 


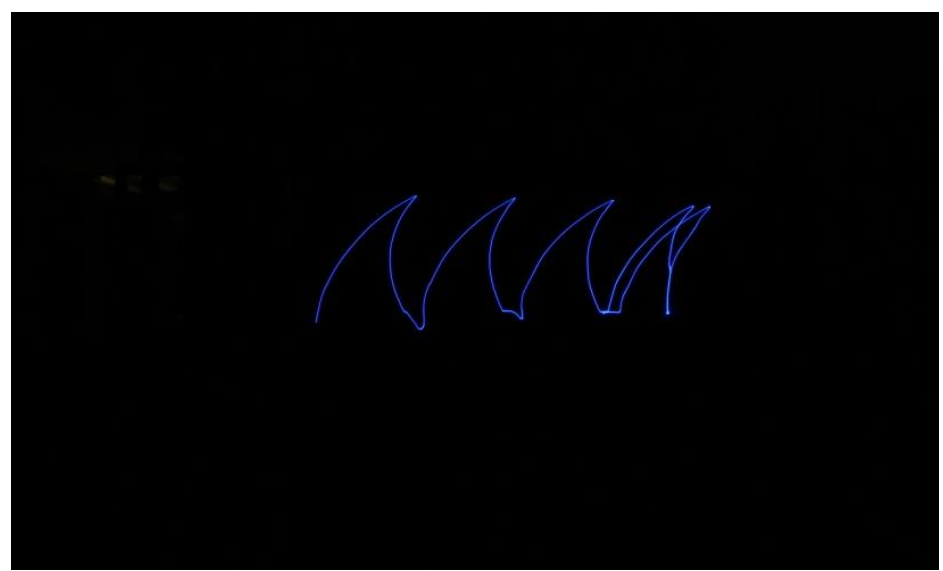

Figure 11. The Pulse-Printing Result of a Participant with the Average Heart Rate of 78

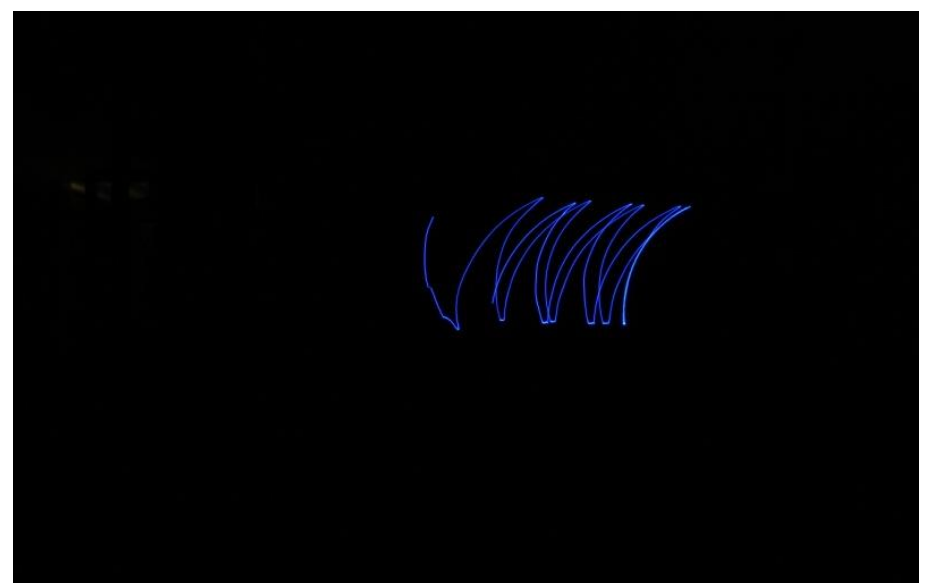

Figure 12. The Pulse-Printing of a Participant with the Average Heart Rate of 105

Figure 11 and Figure 12 show the experimental results of the spectators with low and high heart rates. One is the spectator's graph with the heart rate of 78, and the other is with the heart rate of 105 . These experimental results verify the feasibility of the proposed artwork.

\section{Conclusions}

This paper presented the first step towards the artistic creation of a pulse printer that interacts physically with the spectators; one that is not used for medical purpose but for aesthetic pleasure. Using this artwork, the spectators can feel and watch his/her own emotion in extraplanar presentation. The most difficult step in this procedure was generating an intuitive heart rate. This was achieved and demonstrated via the mapping mechanism. This artwork provides an ideal platform for intuitively and pleasantly storing the spectators' heart rates or emotions in a wide variety of situations through a DSLR camera.

This aesthetic artwork reinvented the expression and usage of the body's signal, and such technique can be used for medical purposes if it is diversely applied. 


\section{Acknowledgements}

This work was supported by the National Research Foundation of Korea Grant funded by the Korean Government (NRF-2013S1A5A8020988).

\section{References}

[1] Afterimage, Available: http://en.wikipedia.org/wiki/Afterimage

[2] M.Z. Poh, D. McDuff and R. Picard, "A Medical Mirror for Non-contact Health Monitoring.", Proceedings of the ACM SIGGRAPH Emerging Technologies, (2011) August 07-11; Vancouver, Canada.

[3] P. Artus and S. Trail, "Proceedings of the ACM SIGGRAPH 2012 Art Gallery", (2012) August 05-09; Los angeles, USA, Available: http://www.philippartus.com/snail/idea.php

[4] L. Hoffman, "Art Review: Light art shines in 'After Image' ", (2003) September; Pittsburgh, USA, Available: http://old.post-gazette.com/ae/20030926wood0926fnp3.asp

[5] V. Henn and D. Straumann, "Three-dimensional eye movement recording for clinical application", Journal of Vestibular Research, 9, 3, (1999).

[6] “The Propeller Clock”, Available: http://www.youtube.com/watch?v=-6JnAxTXApw

[7] M. Ohta and Y. Sako, "LED Display", U.S. Patent 5,444,456A, May 23 (1991).

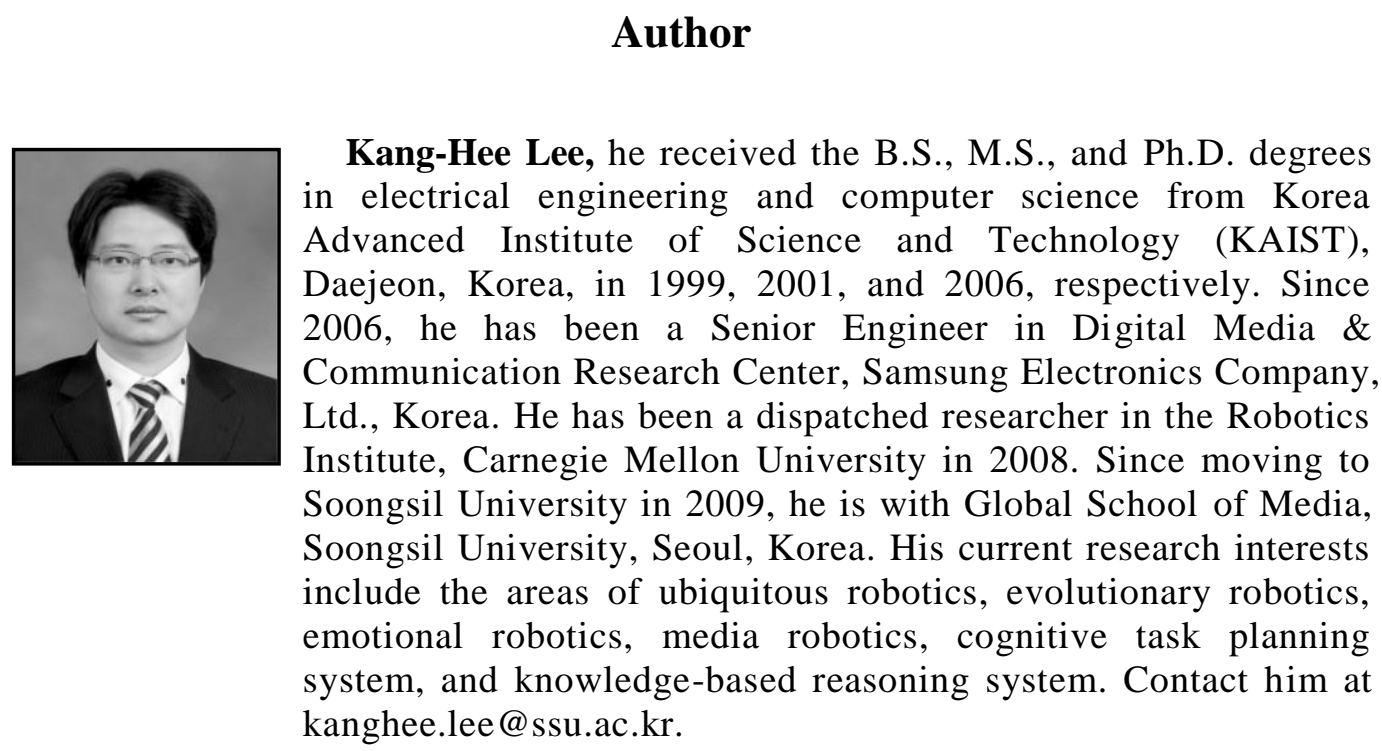

